P164 (continued)

Conclusions and Implications: This program supports child care providers to implement responsive feeding practices with children in their care.

Funding: USDA Nebraska Extension.

\section{P165 Effect of Cooking Classes on Healthy Eating Behavior Among College Students}

Anna Barr, South Dakota State University, Department of Health and Nutritional Sciences; Andrea Hanson, BS; Kendra Kattelmann, PhD, LDN, RDN, FAND, kendra.kattelmann@sdstate.edu, South Dakota State University, Department of Health and Nutritional Sciences, 1015 Campanile Avenue, Brookings, SD 57007

Background (Background, Rationale, Prior Research, and/or Theory): Many college students do not understand how to prepare healthy meals accounting for food safety, which can prompt students to consume convenient, often unhealthy foods.

Objective: To determine if cooking classes tailored to college students would improve knowledge and behavior to eat healthfully and practice food safety.

Study Design, Setting, Participants, Intervention: A one-group, pre- and post-test model was used. Three cooking-classes were developed with parameters of limited cooking skills, limited access to kitchen equipment, and shelf-stable ingredients that are readily available on campuses (i.e. convenience stores and salad bars). Participants $(\mathrm{n}=38)$ were recruited using tabling, posters, email, and social media.

Outcome Measures and Analysis: Pre- and post- surveys assessed nutrition knowledge, frequency of healthy eating, cooking confidence, cooking ability, cooking frequency, and food safety knowledge. Participants were also queried on their perception of the cooking classes and their dietary behaviors post-intervention.

Results: Scores for nutrition knowledge $(P=.000)$, cooking confidence $(P=.026)$, ability to put together ready-made ingredients to make a complete meal $(P=.016)$, and preparing dishes from basic ingredients $(P=.006)$ increased. There were no changes in ability to cook convenience foods, cooking frequency, food safety knowledge, nor frequency of healthy food consumption. However, $83 \%$ of participants perceived that they were consuming healthier diets because of the cooking classes.

Conclusions and Implications: Cooking classes tailored to college students with limited access to cooking facilities, equipment, and ingredients that can be purchased on campus were effective in improving nutrition knowledge, cooking confidence, the ability to put together ready-made ingredients to make a complete meal, and the ability to prepare dishes from basic ingredients. Despite participants' reports that their diets were healthier after the cooking classes; there was no significant change in the frequency of choosing healthier foods detected via survey assessments. Cooking classes tailored to the college student population can be used to educate about nutrition and food safety and improve confidence and cooking ability.
Funding: USDA.

\section{P166 Effects of Fruvapalooza Health Fair: An Evaluation of Intended Health Behaviors}

Sa'Nealdra Wiggins, BS, swiggin8@vols.utk.edu, University of Tennessee, $1215 \mathrm{~W}$ Cumberland Avenue, Knoxville, TN 37996; Sarah Colby, PhD, RDN; Jessica Dietrich, BS; Chelsea Allison, BS; Kristin Riggsbee, BS; Molly Rigell Peek, MA, BA; Marleah Payne, BS

Background (Background, Rationale, Prior Research, and/or Theory): Get Fruved is a multi-state social marketing and environmental change intervention designed to promote healthy behaviors associated with obesity prevention among older adolescents.

Objective: To identify intended health behavior changes reported after attending a health fair event.

Study Design, Setting, Participants, Intervention: As a part of the Get Fruved project, college students mentored high school students in collecting data on student health behaviors. High school students then creatively communicated the findings (oral presentations, posters, songs, children's books, visual art, videos, or interactive exhibits) at a high school health fair called "Fruvapalooza."

Outcome Measures and Analysis: Fruvapalooza attendees were asked to complete anonymous surveys on experiences at the event and any resulting changes in intended health behavior.

Results: Out of approximately 200 attendees, 31 surveys were completed. Most (90\%) rated the event as excellent and $74 \%$ reported that they planned to make a health change because of attending the event. Intended behavior changes included sleeping more, getting more exercise, drinking more water, eating healthier food, and managing stress.

Conclusions and Implications: High school health fairs may be effective in influencing behavioral intentions of attendees. Future research is needed to determine if these changes in intention translates into behavior change.

Funding: NIFA.

\section{P167 Experiential Learning: Community Nutrition Class Field Trip to Rural Community With Associated Program Planning}

Tammy Stephenson,PhD, Tammy.Stephenson@uky.edu, University of Kentucky, Department of Dietetics and Human Nutrition, 203 Funkhouser Building, Lexington, KY 40506-0054; Dawn Brewer, PhD, RDN;

Elizabeth Combs, MS, RDN; Annie Koempel, BS;

Kelci McHugh, BS

Objective: Evaluate the impact of a rural communitybased experiential learning (EL) project and field trip on upper-division nutrition student knowledge and understanding of challenges and opportunities of healthcare professionals working in a rural community.

Continued on page S87 